\title{
Advanced Detector Development for Electron Microscopy Enables New Insight into the Study of the Virus Life Cycle in Cells and Alzheimer's Disease.
}

Anna-Clare Milazzo, Jason Lanman*, James C. Bouwer, Liang Jin, Steven T. Peltier, John E. Johnson*, Stuart Kleinfelder ${ }^{\#}$, Nguyen-huu Xuong, Mark H. Ellisman.

National Center for Microscopy and Imaging Research, Center for Research in Biological Systems, University of California at San Diego, La Jolla, CA 92093

* Department of Molecular Biology, The Scripps Research Institute, La Jolla, CA 92037

\# EECS Department, University of California, Irvine, CA 92697

We have pioneered the development of lens-coupled scintillator based Charge Coupled Device (CCD) detectors resulting in the production of an $8 \mathrm{k} \times 8 \mathrm{k}$ lens-coupled, high-resolution, wide field imaging system [1 and 4] and a direct detection device (DDD), which forgoes the use of a scintillator to produce electron images in a radiation- hardened CMOS device [2-4]. Both of these systems have been used to collect electron microscope tomograms revealing new insights into virus lifecycles and Alzheimer's disease.

The custom designed 8k x 8k lens coupled, high-resolution, wide-field Supra-cam allows us to produce digital images that exceed the format and sensitivity of film. This camera has allowed scientists to view the detailed structure of macro-molecular complexes at the scale of entire cells. With the use of our "Supracam," we have gained new insight into the cellular lifecycle of Flock House virus (FHV) within cells and have used the ultra-wide-field imaging capabilities to image Alzheimer's patient brain tissue. FHV forms large para-crystalline arrays $(\sim 1$ to $4 \mu \mathrm{m})$ inside the cells (Figure 1$)$. Using the Supra-cam, we were able to image entire cells containing the viral arrays. This has revealed the highly ordered lattice that is associated with large bundles of filaments $(\sim 200 \mathrm{~nm})$ within the array. These filament bundles indicate the virus particles are trafficked via a cellular highway from the replication site, within the infected cell's mitochondria to a central location. The viral RNA (vRNA) is replicated by the viral RNA-dependant RNA polymerase (RdRp), which modifies the outer mitochondrial membrane to form spherules. Our hypothesis from the Supra-cam data is that following RNA release from the spherules, ribosomes synthesize virus coat protein, which co-assembles with the RNA to form newly assembled virus particles. Testing this model requires the imaging of wide enough areas to simultaneously capture the newly assembled virus particles (neighboring the modified mitochondria), the trafficking virus particles, and the viral arrays (an area of about $4 \mu \mathrm{m})$. Additionally the images need to be of sufficient quality to discern virus particles (30nm) from the similarly sized ribosomes $(29 \mathrm{~nm})$. All of these requirements are met with the high resolution afforded by the $8 \mathrm{k}$ x 8k system.

The Supra-cam has also been used to capture ultra-wide field images of brain biopsies from Alzheimer's disease patients. By coupling the $8 \mathrm{k} \mathrm{x} 8 \mathrm{k}$ detector with custom designed goniometer stage-control hardware, NCMIR had been able to produce 3.6 billion pixel, 16 bit montaged images and larger that map greater than $150 \mu \mathrm{m} \times 150 \mu \mathrm{m}$ of tissue at a $2 \mathrm{~nm}$ resolution. These ultra-wide field brain maps are advancing our ability to understand how Alzheimer's plaques affect neighboring neurons and the density of synapses (see Figure 1 for a single $8 \mathrm{k} \times \mathrm{k}$ image).

Complementing the use of the Supra-cam, we recently developed a new 1k x 1k analog Direct Detection Device (DDD) that has been used to collect several tomograms of FHV infections in vivo. While the Supracam offers high-resolution, wide-field imaging, the DDD has extremely high sensitivity and small pixel size, making it ideal for higher resolution, lower dose studies. We have performed tomography with the DDD, focusing on the highly ordered FHV arrays in Drosophila cell. Resin-embedded specimens were prepared using high-pressure freezing and freeze substitution, allowing for a high degree of preservation of 
molecular details. By imaging enough particles we can compare conditions staining primarily RNA and DNA to conditions staining the complete virus capsid allowing us to examine the locations of these components during the replications process. As mentioned before, due to the small pixel size of the DDD (5 $\mu \mathrm{m})$, these tomograms were taken at much lower magnification $(\sim 9-15 \mathrm{k})$ compared to a typical magnification of $30-45 \mathrm{kx}$ used with a standard CCD imaging system. The lower magnification translates to a lower dose on the specimen. Additionally, the high signal to noise (SNR 10:1), means that each image in the tilt series was clearly resolved with about 60 electrons/pixel, translating to a very low dose of $\sim 4$ electrons/angstrom ${ }^{2}$. Figure 2 provides an image from a tilt series showing very high contrast despite the low dose. By exploiting these characteristics of the DDD, we aim to conduct higher resolution tomography with high throughput to accelerate our ability to elucidate the virus life cycle within the cell.

1. G. Y. Fan et al., Ultramicroscopy 84 (2000) 75.

2. L. Jin et al., J Struct Biol 161 (2008) 352

3. A. C. Milazzo et al., Ultramicroscopy104 (2005) 152.

4. N. H. Xuong et al., Methods Cell Biol 79 (2007) 721.

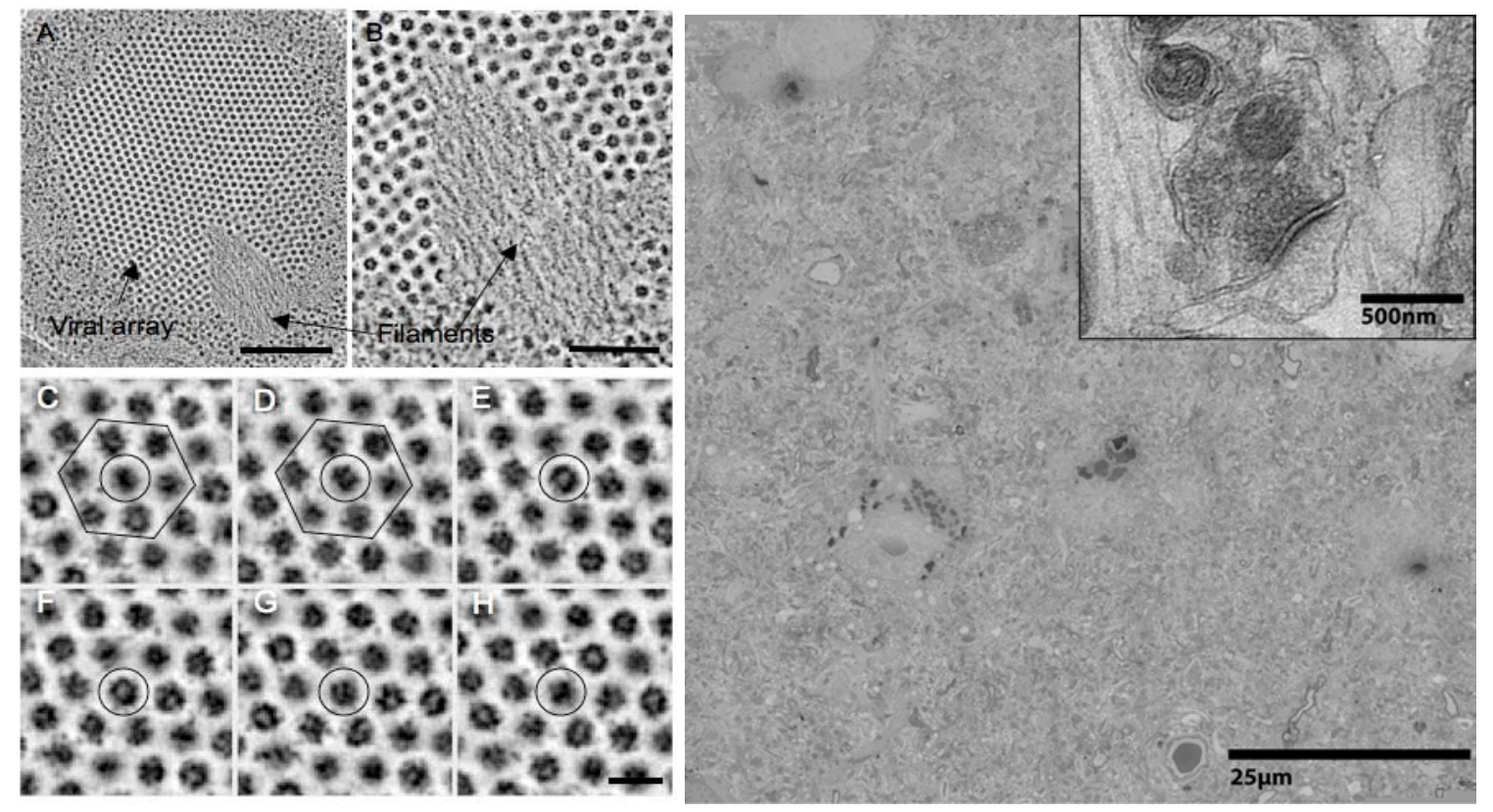

Figure 1. EM images of FHV infected cells and Alzheimer's plaques with the Supra-cam. The panel on the left shows EM tomograms of a FHV array in drosophila cells collected on the Supra-cam. A slice from the tomogram shows the viral array in the cytoplasm (A). A slice at higher magnification demonstrates the association of fibrils with the viral array (B). Six slices are shown at higher magnification $(\mathrm{C}-\mathrm{H})$. The same virus particle observed in $\mathrm{C}-\mathrm{H}$ is circled to indicate the less densely stained core of the virus ( $\mathrm{E}$ and $\mathrm{F}$ ) and the neighboring virus particles in the array are indicated by a hexamer $(\mathrm{C}-\mathrm{H})$. The scale bars are $500 \mathrm{~nm}(\mathrm{~A}), 200 \mathrm{~nm}(\mathrm{~B})$ and $50 \mathrm{~nm}(\mathrm{C}-\mathrm{H})$. The panel on the right shows a 3.6 billion pixel image of an Alzheimer's patient's brain. The inset shows a $1 \mathrm{k} x 1 \mathrm{k}$ sub image showing a synapse, synaptic vesicles, and mitochondria at $2 \mathrm{~nm}$ resolution.
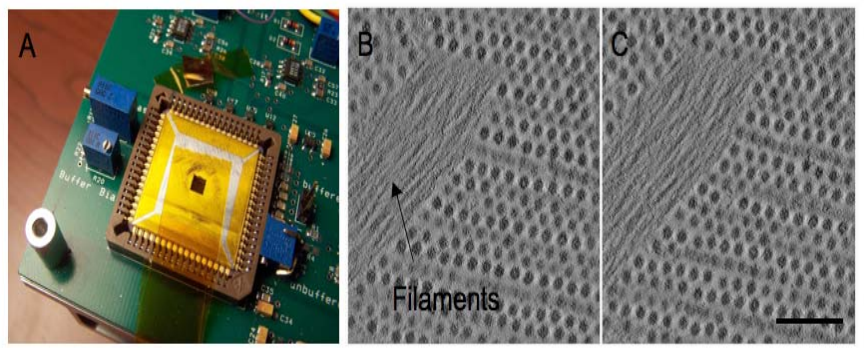

Figure 2. Tomogram collected on DDD. The DDD that was used to collect the EM tomograms (A). Slices from a single tilt tomogram of a FHV array in Drosophila cells. The scale bar is $200 \mathrm{~nm}$. 